\title{
Applications of Homomorphism on the Structure of Semigroups \\ Applications of Homomorphism on the Structure
}

\author{
Huishu Yuan, Xiangzhi Kong \\ School of Science, Jiangnan University, Wuxi, China \\ Email: xiangzhikong@jiangnan.edu.cn
}

Received December 4, 2013; revised January 4, 2014; accepted January 10, 2014

Copyright (C) 2014 Huishu Yuan, Xiangzhi Kong. This is an open access article distributed under the Creative Commons Attribution License, which permits unrestricted use, distribution, and reproduction in any medium, provided the original work is properly cited. In accordance of the Creative Commons Attribution License all Copyrights (C) 2014 are reserved for SCIRP and the owner of the intellectual property Hui shu Yuan, Xiang zhi Kong. All Copyright @ 2014 are guarded by law and by SCIRP as a guardian.

\section{ABSTRACT}

By utilizing homomorphisms and $G$-strong semilattice of semigroups, we show that the Green $(*, \sim)$-relation $\mathcal{H}^{*, \sim}$ is a regular band congruence on a $r$-ample semigroup if and only if it is a $G$-strong semilattice of completely $\mathcal{J}^{*, \sim}$-simple semigroups. The result generalizes Petrich's result on completely regular semigroups with Green's relation $\mathcal{H}$ a normal band congruence or a regular band congruence from the round of regular semigroups to the round of $r$-ample semigroups.

\section{KEYWORDS}

\section{Homomorphism; Natural Partial Order; Green's $(*, \sim)$-Relation; Semilattice Decomposition}

\section{Introduction and Preliminaries}

It is well known that the usual Green's relations on a semigroup $S$ play an important role in the study of the structure of regular semigroups [1-7]. Especially, the well-known theorem of A. H. Clifford states that a semigroup is a completely regular semigroup if and only if it can be expressed as a semilattice of completely simple semigroups (see [1]), where a completely regular semigroup is a semigroup whose $\mathcal{H}$-class contains an idempotent. By using this result, A. H. Clifford, M. Petrich both showed that a completely regular semigroup $S$ with its Green's relation $\mathcal{H}$ is a normal band congruence if and only if $S$ is a strong semilattice of complete simple semigroups [3]. On the other hand, J. B. Fountain generalized the Clifford theorem by showing that an abundant semigroup is a superabundant semigroup, that is, an abundant semigroup $S$ with every $\mathcal{H}^{*}$-class of $S$ contains an idempotent of $S$ if and only if $S$ is a semilattice of completely $\mathcal{J}^{*}$-simple semigroups.

We first recall some of the generalized Green's relations which are frequently used to study the structure of abundant semigroups. The following Green *-relations on a semigroup $S$ were originally due to F. Pastijn [8] and were extensively used by J. B. Fountain to study the so called abundant semigroups in [9]. Let $S$ be an arbitrary semigroup. Then, we define $\mathcal{L}^{*}=\left\{(a, b) \in S \times S:\left(\forall x, y \in S^{1}\right) a x=a y \Leftrightarrow b x=b y\right\}$. Dually, we define $\mathcal{R}^{*}=\left\{(a, b) \in S \times S:\left(\forall x, y \in S^{1}\right) x a=y a \Leftrightarrow x b=y b\right\}$ and define $\mathcal{H}^{*}=\mathcal{L}^{*} \cap \mathcal{R}^{*}, \mathcal{D}^{*}=\mathcal{L}^{*} \vee \mathcal{R}^{*}$, while $\mathcal{J}^{*}=\left\{(a, b) \in S \times S: J^{*}(a)=J^{*}(b)\right\}$, where $J^{*}(a)$ is the smallest ideal containing element a saturated by $\mathcal{L}^{*}$ and $\mathcal{R}^{*}$, that is, $J^{*}(a)$ is a union of some $\mathcal{L}^{*}$-classes and also a union of some $\mathcal{R}^{*}$-classes of $S$.

It was given by M. V. Lawson in [10] the definition of $\tilde{\mathcal{R}}$ on a semigroup $S$ as 
$a \tilde{\mathcal{R}} b \Leftrightarrow(\forall e \in E(S)) e a=a \leftrightarrow e b=b$, where $E(S)$ is the idempotents set of $S$. It can be easily seen that $\mathcal{R}^{*} \subseteq \tilde{\mathcal{R}}$ and for any regular elements $a, b$ of a semigroup $S, a \mathcal{R} b$ if and only if $a \tilde{\mathcal{R}} b$.

In order to further investigate the structure of non-regular semigroups, we have to generalize the usual Green's relations. For this purpose, J. B. Fountain and F. Pastijn both generalized the Green's relations to the so called Green * *-relations in [8] and [9], respectively and by using these Green * *-relations, many new results of rpp -semigroups and abundant semigroups have been obtained by many authors in [10-18]. For the results of all other generalized Green's relations and their mutual relationships, the reader is referred to a recent paper of Shum, Du and Guo [17].

In this paper, we introduce the concept of the Green $(*, \sim)$-relations which is a common generalization of the Green *-relations and the $\tilde{\mathcal{R}}$ relation. We also introduce the concept of the $G$-strong semilattice of semigroups and give the semilattice decomposition of a $r$-ample semigroup whose $\mathcal{H}^{*, \sim}$ is a congruence. By using this decomposition, we will show that a semigroup $S$ is a $r$-ample semigroup whose $\mathcal{H}^{*, \sim}$ is a regular band congruence if and only if $S$ is a $G$-strong semilattice of completely $\mathcal{J}^{*, \sim}$-simple semigroups. Our result extends and enriches the results of A. H. Clifford, M. Petrich and J. B. Fountain in the literature.

We first generalize the usual Green's relations and the Green *-relations to the Green $(*, \sim)$-relations on a semigroup $S$.

$$
\begin{aligned}
& \mathcal{L}^{*, \sim}=\mathcal{L}^{*}, \mathcal{R}^{*, \sim}=\tilde{R} \\
& \mathcal{H}^{*, \sim}=\mathcal{L}^{*, \sim} \cap \mathcal{R}^{*, \sim}, \mathcal{D}^{*, \sim}=\mathcal{L}^{*, \sim} \vee \mathcal{R}^{*, \sim} \\
& \mathcal{J}^{*, \sim}=\left\{(a, b) \in S \times S: J^{*, \sim}(a)=J^{*, \sim}(b)\right\} .
\end{aligned}
$$

where $J^{*, \sim}(a)$ is the smallest ideal containing a saturated by $\mathcal{L}^{*, \sim}$ and $\mathcal{R}^{*, \sim}$. We can easily see that $\mathcal{L}^{*, \sim}$ is a right congruence on $S$ while $\mathcal{R}^{*, \sim}$ is only an equivalence relation on $S$. One can immediately see that there is at most one idempotent contained in each $\mathcal{H}^{*, \sim}$-class. If $e \in H_{a}^{*, \sim} \cap E(S)$, for some $a \in S$, then we write $e$ as $x^{0}$, for any $x \in H_{a}^{*, \sim}$. Clearly, for any $x \in H_{a}^{*, \sim}$ with $a \in S$, we have $x=x x^{0}=x^{0} x$.

If a semigroup $S$ is a regular semigroup, then every $\mathcal{L}$-class of $S$ contains at least one idempotent, and so does every $\mathcal{R}$-class of $S$. If $S$ is a completely regular semigroup, then every $\mathcal{H}$-class of $S$ contains an idempotent, in such a case, every $\mathcal{H}$-class is a group. A semigroup $S$ is called an abundant semigroup by J. B. Fountain in [9] if every $\mathcal{L}^{*}$ - and $\mathcal{R}^{*}$-class of $S$ contains an idempotent. One can easily see that $\mathcal{L}^{*}=\mathcal{L}$ on the regular elements of a semigroup. Therefore, all regular semigroups are obviously abundant semigroups. As an analogy of the orthodox subsemigroup in a regular semigroup, a subsemigroup in an abundant semigroup is called a superabundant semigroup [9] if each of the $\mathcal{H}^{*}$-classes of the abundant semigroup $S$ contains an idempotent, in such a case, every $\mathcal{H}^{*}$-class of $S$ is a cancellative monoid, which is the generalization of completely regular semigroups within the classes of abundant semigroups. The concept of ample semigroups was first mentioned in the paper of G. Gomes and V. Gould [19]. We now call a semigroup $r$-ample if each $\mathcal{L}^{*, \sim}$ class and each $\mathcal{R}^{*, \sim}$-class contain an idempotent, the concept was first mentioned by Y. Q. Guo, K. P. Shum and C. M. Gong [3]. Certainly, an abundant semigroup is a $r$-ample semigroup, but the converse is not true, and an example can be found in [3]. We now call a semigroup $S$ a super $r$-ample semigroup if each $\mathcal{H}^{*, \sim}$ class of $S$ contains an idempotent and $\mathcal{H}^{*, \sim}$ is a congruence. It is clear that every $\mathcal{H}^{*, \sim}$-class of such super $r$-ample semigroup forms a left cancellative monoid which is a generalization of the completely regular semigroups and the superabundant semigroups in the classes of $r$-ample semigroups.

It is recalled that a regular band is a band that satisfies the identity axya=axaya. For further notations and terminology, such as strong semilattice decomposition of semigroups, the readers refer to [2,3]. For some other concepts that have already appeared in the literature, we occasionally use its alternatives, though equivalent definitions.

\section{Properties of r-Ample Semigroups}

A completely simple semigroup is a $\mathcal{J}$-simple completely regular semigroup whose Green's relation $\mathcal{H}$ is a congruence on $S$, as a natural generalization of this concept, we call a $r$-ample semigroup $S$ a completely $\mathcal{J}^{*, \sim}$-simple semigroup if it is a $\mathcal{J}^{*, \sim}$-simple semigroup and the Green $(*, \sim)$-relation $\mathcal{H}^{*, \sim}$ is a congruence on $S$.

We first state the following crucial lemma. 
Lemma 1 Let $S$ be a $r$-ample semigroup with each $\mathcal{H}^{*, \sim}$-class contains an idempotent. Then the Green $\mathcal{H}^{*, \sim}$ relation on $S$ is a congruence on $S$ if and only if for any $a, b \in S,(a b)^{0}=\left(a^{0} b^{0}\right)^{0}$.

Proof. Necessity. Let $a, b \in S$. Then, $a \mathcal{H}^{*, \sim} a^{0}$ and $b \mathcal{H}^{*, \sim} b^{0}$. Since $\mathcal{H}^{*, \sim}$ is a congruence on $S$, $a b \mathcal{H}^{*, \sim} a^{0} b^{0}$. But $a b \mathcal{H}^{*, \sim}(a b)^{0}$ and so, $(a b)^{0}=\left(a^{0} b^{0}\right)^{0}$ since every $\mathcal{H}^{*, \sim}$-class contains a unique idempotent.

Sufficiency. Since $\mathcal{H}^{*, \sim}$ is an equivalence on $S$, we only need to show that $\mathcal{H}^{*, \sim}$ is compatible with the multiplication of $S$. Let $(a, b) \in \mathcal{H}^{*, \sim}$ and $c \in S$. Then $(c a)^{0}=\left(c^{0} a^{0}\right)^{0}=\left(c^{0} b^{0}\right)^{0}=(c b)^{0}$ and so that $\mathcal{H}^{*, \sim}$ is left compatible with the multiplication on $S$. Similarly, $\mathcal{H}^{*, \sim}$ is right compatible wit the multipication on $S$ and thus $\mathcal{H}^{*, \sim}$ is a congruence on $S$.

Lemma 2 If $e, f$ are $\mathcal{D}^{*, \sim}$-related idempotents of a $r$-ample semigroup $S$ with each $\mathcal{H}^{*, \sim}$-class contains an idempotent, then eDf .

Proof. Since $e \mathcal{D}^{*, \sim} f$, there are elements $a_{1}, \cdots, a_{k}$ of $S$ such that

$$
e \mathcal{L}^{*, \sim} a_{1} \mathcal{R}^{*, \sim} a_{2} \cdots a_{k} \mathcal{L}^{*,} f \text {. }
$$

Since $S$ is $r$-ample, $e \mathcal{L}^{*, \sim} a_{1}^{0} \mathcal{L}^{*, \sim} a_{2}^{0} \cdots a_{k}^{0} \mathcal{L}^{*, \sim} f$. Thus, eDf since for regular elements $\mathcal{R}=\mathcal{R}^{*}=\tilde{\mathcal{R}}$ and $\mathcal{L}=\mathcal{L}^{*}$.

Corollary 3 If $S$ is a $r$-ample semigroup with each $\mathcal{H}^{*, \sim}$-class contains an idempotent, then

$$
\mathcal{D}^{*, \sim}=\mathcal{L}^{*, \sim} \circ \mathcal{R}^{*, \sim}=\mathcal{R}^{*, \sim} \circ \mathcal{L}^{*, \sim} \text {. }
$$

Proof. Let $a, b \in S$ and $a \mathcal{D}^{*, \sim} b$. Then, by Lemma 2, $a^{0} \mathcal{D} b^{0}$. Thus, there exist elements $c, d$ in $S$ with $a^{0} \mathcal{L} c \mathcal{R} b^{0}$ and $a^{0} \mathcal{R} d \mathcal{L} b^{0}$. Then $a \mathcal{L}^{*, \sim} c \mathcal{R}^{*, \sim} b$ and $a \mathcal{R}^{*, \sim} d \mathcal{L}^{*, \sim} b$ and the result follows.

Lemma 4 Let $e, f$ be idempotents in a $r$-ample semigroup $S$ with each $\mathcal{H}^{*, \sim}$-class contains an idempotent. if eJf, then eDf.

Proof. Since $S e S=S f S$, there are elements $x, y, s, t$ in $S$ such that $f=s e t, e=x f y$. Let $h=(f y)^{0}$ and $k=(s e)^{0}$. Then $h f y=f y=f f y$ so that $h=h^{2}=f h$, and sek $=s e=$ see so that $k=k^{2}=k e$. It follows that $h f, e k$ are idempotents with $h f \mathcal{R} h$ and $e k \mathcal{L} k$. Hence ehf $\mathcal{R} e h$ and $e k f \mathcal{L} k f$. Now $e h=x f y h=x f y=e$ and $k f=k s e t=s e t=f$ so that $e \mathcal{R e f} \mathcal{L} f$, that is, $e \mathcal{D} f$.

Proposition 5 If $a$ is an element of a $r$-ample semigroup $S$, then $J^{*, \sim}(a)=S a^{0} S$.

Proof. Certainly, $a^{0} \in \mathcal{J}^{*, \sim}(a)$ so that $S a^{0} S \subseteq \mathcal{J}^{*, \sim}(a)$. We now show that the ideal $S a^{0} S$ is actually an ideal which is saturated by $\mathcal{L}^{*, \sim}$ and $\mathcal{R}^{*, \sim}$, since $a=a a^{0} \in S a^{0} S$, the result follows. Let $b=x a^{0} y \in S a^{0} S(x, y \in S)$ and $k=\left(a^{0} y\right)^{0}$. Then $a^{0} a^{0} y=k a^{0} y$ so that $a^{0}\left(a^{0} y\right)^{0}=k^{2}=k$. Also since $\mathcal{H}^{*, \sim}$ is a congruence on $S, x a^{0} y \mathcal{H}^{*, \sim} x k$. Now let $h=(x k)^{0}=\left(x a^{0} y\right)^{0}$. Then $x k h=x k k$ so that $h=h^{2}=h k=h a^{0} k \in S a^{0} S$. Hence if $c \in L_{b}^{*, \sim}, d \in R_{b}^{*, \sim}$, then $c=c h, d=h d \in S a^{0} S$ so that $S a^{0} S$ is indeed an ideal saturated by $\mathcal{L}^{*, \sim}$ and $\mathcal{R}^{*, \sim}$, as required.

Proposition 6 On a completely $\mathcal{J}^{*, \sim}$-simple semigroup $S, \mathcal{J}^{*, \sim}=\mathcal{D}^{*, \sim}$.

Proof. Suppose that $a, b \in S$ with $a \mathcal{J}^{*, \sim} b$. Then, by Proposition 5, $S a^{0} S=S b^{0} S$. By Lemma 4, $a^{0} \mathcal{D} b^{0}$ and so $a \mathcal{H}^{*, \sim} a^{0} \mathcal{D} b^{0} \mathcal{H}^{*, \sim} b$, which implies that $a \mathcal{D}^{*, \sim} b$ and hence $\mathcal{J}^{*, \sim} \subseteq \mathcal{D}^{*, \sim}$. Conversely, let $a, b \in S$ with $a \mathcal{D}^{*, \sim} b$. Now, by Corollary 3 , there exists $c \in S$ such that $a \mathcal{L}^{*, \sim} c \mathcal{R}^{*, \sim} b$. Thus $a^{0} \mathcal{L} c^{0} \mathcal{R} b^{0}$ and so $S a^{0} S=S c^{0} S=S b^{0} S$. By Proposition 5, $(a, b) \in \mathcal{J}^{*, \sim}$ and hence $\mathcal{D}^{*, \sim} \subseteq \mathcal{J}^{*, \sim}$. Now we have $\mathcal{J}^{*, \sim}=\mathcal{D}^{*, \sim}$.

Proposition 7 A completely $\mathcal{J}^{*, \sim}$-simple $S$ is primitive for idempotents.

Proof. Let $e, f$ be idempotents in $S$ with $e \leq f$. Since $S$ is a completely $\mathcal{J}^{*, \sim}$-simple semigroup, it follows from Proposition 5 that $f \in S e S$. Now by the first part of Exercise 3 of $[1, \S 8.4]$ there is an idempotent $g$ of $S$ such that $f \mathcal{D} g$ and $g \leq e$. Let $a \in S$ be such that $f \mathcal{L a R} g$. Then $f \mathcal{L} a^{0} \mathcal{R} g$ and since $g \leq f$ we have

$$
a^{0}=g a^{0}(g f) a^{0}=g\left(f a^{0}\right)=g f=g .
$$


Now we have $g \leq f$ and $g \mathcal{L} f$ and so $f=f g=g$. But $g \leq e$ so that $e=f$ and all idempotent of $S$ are primitive.

Lemma 8 In a completely $\mathcal{J}^{*, \sim}$-simple semigroupm $S$, the regular elements of $S$ generate a completely simple subsemigroup.

Proof. Let $a, b$ be regular elements of $S$. Since $S$ consists of a single $\mathcal{D}^{*, \sim}$-class(by Proposition 6), it follows from Corollary 3 that there is an element $c \in S$ with $a \mathcal{L}^{*, \sim} c \mathcal{R}^{*, \sim} b$. Hence, we have $a \mathcal{L}^{*, \sim} c^{0} \mathcal{R}^{*, \sim} b$. Thus, $c^{0} b=b$ and $a \mathcal{L} c^{0}$ since $a$ is regular. Now we see that $a b \mathcal{L} b$ and the regularity of $a b$ follows from that of $b$. The property of completely simple of the subsemigroup generated by regular elements follows Proposition 6, lemma 2 and Corollary 3 easily.

Theorem 9 Let $S$ be a $r$-ample semigroup.Then $S$ is a semilattice $Y$ of completely $\mathcal{J}^{*, \sim}$-simple semigroups $S_{\alpha}(\alpha \in Y)$ such that for $\alpha \in Y$ and $a \in S_{\alpha}, \quad \mathcal{L}_{a}^{*, \sim}(S)=\mathcal{L}_{a}^{*, \sim}\left(S_{\alpha}\right), \mathcal{R}_{a}^{*, \sim}(S)=\mathcal{R}_{a}^{*, \sim}\left(S_{\alpha}\right)$.

Proof. If $a \in S$, then $a \mathcal{H}^{*, \sim} a^{2}$ so that by Proposition 5, $J^{*, \sim}(a)=J^{*, \sim}\left(a^{2}\right)$. Now for $a, b \in S$, $(a b)^{2} \in S b a S$, and so

$$
J^{*, \sim}(a b)=J^{*, \sim}\left((a b)^{2}\right) \subseteq J^{*, \sim}(b a)
$$

Now, by symmetry, we get $J^{*, \sim}(a b)=J^{*, \sim}(b a)$. By Proposition 5, $J^{*, \sim}(a)=S a^{0} S, J^{*, \sim}(b)=S b^{0} S$ so that if $c \in J^{*, \sim}(a) \cap J^{*, \sim}(b)$, we have $c=x a^{0} y=z b^{0} t$ for some $x, y, z, t \in S$. Now $c^{2}=z b^{0} t x a^{0} y \in S b^{0} t x a^{0} S \subseteq J^{*, \sim}\left(b^{0} t x a^{0}\right)$ and $J^{*, \sim}\left(b^{0} t x a^{0}\right)=J^{*, \sim}\left(a^{0} b^{0} t x\right)$ and by the preceding paragraph. we have $c^{2} \in J^{*, \sim}\left(a^{0} b^{0}\right)$ and since $c \mathcal{H}^{*, \sim} c^{2}, c \in J^{*, \sim}\left(a^{0} b^{0}\right)$. Since $a \mathcal{H}^{*, \sim} a^{0}, b \mathcal{H}^{*, \sim} b^{0}$ and $\mathcal{H}^{*, \sim}$ is a congruence on $S$, and so $a b \mathcal{H}^{*, \sim} a^{0} b^{0}$. Now, $c \in \mathcal{H}^{*, \sim}(a b)$, we have $J^{*, \sim}(a) \cap J^{*, \sim}(b) \subseteq J^{*, \sim}(a b)$ and since the opposite inclusion is clear, we conclude that $J^{*, \sim}(a) \cap J^{*, \sim}(b)=J^{*, \sim}(a b)$.

Because the set $Y$ of all ideals $J^{*, \sim}(a)(a \in S)$ forms a semilattice under the usual set intersection and that the map $a \mapsto J^{*, \sim}(a)$ is a homomorphism from $S$ onto $Y$. The inverse image of $J^{*, \sim}(a)$ is just the $\mathcal{J}^{*, \sim}$ -class $J_{a}^{*, \sim}$ which is thus a subsemigroup of $S$. Hence $S$ is a semilattice $Y$ of the semigroups $J_{a}^{*, \sim}$.

Now let $a, b$ be elements of $\mathcal{J}^{*, \sim}$-class $J^{*, \sim}$ and suppose that $(a, b) \in \mathcal{L}^{*, \sim}\left(J^{*, \sim}\right)$. Certainly $a^{0}, b^{0} \in J^{*, \sim}$ so that we have $\left(a^{0}, b^{0}\right) \in \mathcal{L}^{*, \sim}\left(J^{*, \sim}\right)$, that is, $a^{0} b^{0}=a^{0}, b^{0} a^{0}=b^{0}$ and $\left(a^{0}, b^{0}\right) \in \mathcal{L}^{*, \sim}(S)$. It follows that $(a, b) \in \mathcal{L}^{*, \sim}(S)$ and consequently, since $L_{a}^{*, \sim}(S) \subseteq J^{*, \sim}$, we have $L_{a}^{*, \sim}(S)=L_{a}^{*, \sim}\left(J^{*, \sim}\right)$. A similar argument shows that $R_{a}^{*, \sim}(S)=R_{a}^{*, \sim}\left(J^{*, \sim}\right)$.

From the last paragraph, we have $H_{a}^{*, \sim}\left(J^{*, \sim}\right)=H_{a}^{*, \sim}(S)$ so that $J^{*, \sim}$ is a $r$-ample semigroup.

Furthermore, if $a, b \in J^{*, \sim}$, then by Proposition 6, $(a, b) \in \mathcal{D}^{*, \sim}(S)$ so that, by Corollary 3, there is an element $c$ in $L_{a}^{*, \sim}(S) \cap R_{b}^{*, \sim}(S)=L_{a}^{*, \sim}\left(J^{*, \sim}\right) \cap R_{b}^{*, \sim}\left(J^{*, \sim}\right)$. Thus, $a, b$ are $\mathcal{D}^{*, \sim}$-related in $J^{*, \sim}$ so that $J^{*, \sim}$ is a $\mathcal{J}^{*, \sim}$-simple semigroup.

We need the following crucial lemma.

Lemma 10 Let $S=\left(Y ; S_{\alpha}\right)$ be a $r$-ample semigroup.

1) Let $a \in S_{\alpha}$ and $\alpha \geq \beta$. Then, there exists $b \in S_{\beta}$ with $a \geq b$;

2) Let $a, b, c \in S, b \mathcal{H}^{*, \sim} c$ and $a \geq b, c$. Then, $b=c$;

3) Let $a \in E(S)$ and $b \in S$ be such that $a \geq b$. Then, $b \in E(S)$.

Proof. 1) Let $b \in S_{\beta}$. Then, by Lemma $1, a(a b a)^{0},(a b a)^{0} a$ and $(a b a)^{0}$ are in the same $\mathcal{H}^{*, \sim}$-class and so $a(a b a)^{0}=(a b a)^{0} a(a b a)^{0}=(a b a)^{0} a$. Let $b=a(a b a)^{0}$. Then $b \in S_{\beta}$ and $a \geq b$.

2) By the definition of " $\geq$ ", there exist $e, f, g, h \in E(S)$ such that $b=e a=a f, c=g a=a h$. From $e b=b$ and $b \mathcal{H}^{*, \sim} b^{0}$, we have $e b^{0}=b^{0}$. Similarly, $c^{0} h=c^{0}$. Thus, $e c=e c^{0} c=e b^{0} c=b^{0} c=c$. Similarly, $b h=b$ and so $b=b h=e a h=e c=c$, as required.

3) We have $b=e a=a f$ for some $e, f \in E(S)$ whence 


$$
b^{2}=(e a)(a f)=e a^{2} f=b .
$$

Following Proposition 7, we can easily prove the following lemma

Lemma 11 Let $\phi$ be a homomorphism from a completely $\mathcal{J}^{*, \sim}$-simple semigroup $S$ into another completely $\mathcal{J}^{*, \sim}$-simple semigroup $T$. Then $(a \phi)^{0}=a^{0} \phi$.

If $\phi$ is a homomorphism between two completely $\mathcal{J}^{*, \sim}$-simple semigroups. Then the Green $(*, \sim)$ -relations $\mathcal{L}^{*, \sim}, \mathcal{R}^{*, \sim}$ are preserved, so that $\mathcal{D}^{*, \sim}$ is preserved. We call a homomorphism preserving $\mathcal{L}^{*, \sim}$, $\mathcal{R}^{*, \sim}$ are good. By Proposition 7 and Lemma 10, we can show that a completely $\mathcal{J}^{*, \sim}$-simple semigroup is primitive.

\section{G-Strong Semilattice Structure of r-Ample Semigroups}

In this section, we introduce the $G$-strong semilattice of semigroups which is a generalization of the well known strong semilattice of semigroups.

Definition 12 Let $S=\left(Y ; S_{\alpha}\right)$ be a semilattice $Y$ decomposition of semigroup $S$ into subsemigroups $S_{\alpha}(\alpha \in Y)$. Suppose that the following conditions hold in the semigroup $S$.

(C1) for any $\alpha, \beta \in Y$, there is a band congruence $\rho_{\alpha, \beta}$ on $S_{\beta}$ with congruence classes $\left\{S_{d(\alpha, \beta)}: d(\alpha, \beta) \in D(\alpha, \beta)\right\}$, where $D(\alpha, \beta)$ is the index set and for $\alpha \in Y, \rho_{\alpha, \alpha}$ is the universal relation $\omega_{S_{\alpha}}$;

(C2) for $\alpha \geq \beta$ on $Y$ and any $d(\alpha, \beta) \in D(\alpha, \beta)$, there is a homomorphism $\phi_{d(\alpha, \beta)}$ from $S_{\alpha}$ into $S_{d(\alpha, \beta)}$. Let $\Phi_{\alpha, \beta}=\left\{\phi_{d(\alpha, \beta)}: d(\alpha, \beta) \in D(\alpha, \beta)\right\}$. Then

1) for $\alpha \in Y$, the homomorphism $\phi_{D(\alpha, \alpha)}: S_{\alpha} \rightarrow S_{\alpha}$ is the identity automorphism of the semigroup $S_{\alpha}$.

2) for $\alpha \geq \beta \geq \gamma$ on $Y, \Phi_{\alpha, \beta} \Phi_{\beta, \gamma} \subseteq \Phi_{\alpha, \gamma}$, where $\Phi_{\alpha, \beta} \Phi_{\beta, \gamma}$ is the set

$\left\{\phi_{d(\alpha, \beta)} \phi_{d(\beta, \gamma)}: d(\alpha, \beta) \in D(\alpha, \beta), d(\beta, \gamma) \in D(\beta, \gamma)\right\}$.

3) for $b \in S_{\beta}$, there exists $\phi_{d(\alpha, \alpha \beta)}^{b} \in \Phi_{\alpha, \alpha \beta}$, for all $a \in S_{\alpha}$,

$$
a b=\left(a \phi_{d(\alpha, \alpha \beta)}^{b}\right)\left(b \phi_{d(\beta, \alpha \beta)}^{a}\right) .
$$

If the semigroup $S$ satisfies the above conditions, then we call $S$ a $G$-strong semilattice of subsemigroups $S_{\alpha}(\alpha \in Y)$ and write $S=G\left[Y ; S_{\alpha}, \Phi_{\alpha, \beta}\right]$. One can easily see that a $G$-strong semilattice $S=G\left[Y ; S_{\alpha}, \Phi_{\alpha, \beta}\right]$ is the ural strong semilattice if and only if all $|D(\alpha, \beta)|=1$ for all $\alpha \geq \beta$ on $Y$.

Following Theorem 9, we can easily see that a $r$-ample semigroup $S$ is a semilattice of completely $\mathcal{J}^{*, \sim}$ simple semigroups $S_{\alpha}(\alpha \in Y)$. In this section, we introduce the band congruence $\rho_{\alpha, \beta}$ on a regular $r$-ample semigroup $S=\left(Y ; S_{\alpha}\right)$ and the structure homomorphisms set $\Phi_{\alpha, \beta}$. Finally, we will show the main result of the paper, that is, a $r$-ample semigroup is a regular $r$-ample semigroup if and only if it is a $G$-strong semilattice of completely $\mathcal{J}^{*, \sim}$-simple semigroups.

Lemma 13 Let $S=\left(Y ; S_{\alpha}\right)$ be a regular $r$-ample semigroup, that is, $S$ is a $r$-ample semigroup with the Green $(*, \sim)$-relation $\mathcal{H}^{*, \sim}$ as a regular band congruence on $S$. Then, for any element $\alpha, \beta \in Y$, we define $\rho_{\alpha, \beta}$ on $S_{\beta}$ as the following:

$$
\left(x, y \in S_{\beta}\right)(x, y) \in \rho_{\alpha, \beta} \Leftrightarrow(a x a)^{0}=(\text { aya })^{0}
$$

for some $a \in S_{\alpha}$. Then

1) $\rho_{\alpha, \beta}$ is a band congruence on $S_{\beta}$ and for $x, y \in S_{\beta},(x, y) \in \rho_{\alpha, \beta}$ if and only if for any $b \in S_{\alpha}$, $(b x b)^{0}=(b y b)^{0}$.

2) for $\alpha \geq \beta \geq \gamma$ on $Y, \rho_{\alpha, \gamma} \subseteq \rho_{\beta, \gamma}$ and $\rho_{\alpha, \alpha}$ is the universal relation $\omega_{S_{\alpha}}$ on $S_{\alpha}$. 
3) for $\alpha \geq \beta$ on $Y$ and $a \in S_{\alpha}, b \in S_{\beta}, a b \rho_{\alpha, \beta} b \rho_{\alpha, \beta} b a$.

Proof. We only prove 1), 2) and 3) can be proved similarly. Let $x, y \in S_{\beta}$ with $(x, y) \in \rho_{\alpha, \beta}$, then there exists $a \in S_{\alpha}$ such that $(a x a)^{0}=(a y a)^{0}$. For any element $b \in S_{\alpha}$, we have $b(a x a)^{0} b=b(a y a)^{0} b$. Thus $\left(b(a x a)^{0} b\right)^{0}=\left(b(a y a)^{0} b\right)^{0}$. By the property of regular bands and Lemma 1 and Lemma 8 , we easily have $(b x b)^{0}=(b y b)^{0}$. Now the proof is completed.

We denote the $\rho_{\alpha, \beta}$-congruence classes by $\left\{S_{d(\alpha, \beta)}: d(\alpha, \beta) \in D(\alpha, \beta)\right\}$, following Lemma 13, $D(\alpha, \alpha)$ is a singleton.

Lemma 14 Let $S=\left(Y ; S_{\alpha}\right)$ be a regular $r$-ample semigroup.

1) For any $\alpha \geq \beta$ on $Y$ and $d(\alpha, \beta) \in D(\alpha, \beta)$. Let $a \in S_{\alpha}$, there exists a unique element $a_{d(\alpha, \beta)} \in S_{d(\alpha, \beta)}$ such that $a \geq a_{d(\alpha, \beta)}$.

2) For any $\alpha \geq \beta$ on $Y$ and $a \in S_{\alpha}, \quad x \in S_{d(\alpha, \beta)}$, if $a^{0} \geq e$ for some idempotent $e \in S_{d(\alpha, \beta)}$, then $e a x=a x, x a e=x a, e a=a e$ and $(e a)^{0}=e$.

Proof. 1) By Lemma 132 ) and Lemma 101$)$, for any $c \in S_{d(\alpha, \beta)}$, the element $a_{d(\alpha, \beta)}=a(a c a)^{0}=(a c a)^{0} a \in S_{d(\alpha, \beta)}$ such that $a \geq a_{d(\alpha, \beta)}$. Easily see $a_{d(\alpha, \beta)}^{0}=(a c a)^{0}$. If there is another $b \in S_{d(\alpha, \beta)}$ such that $a \geq b$, then there are idempotents $g, h \in E(S)$ such that $b=g a=a h$ and so $b a^{0}=b=a^{0} b$, thus $b^{0} a^{0}=b^{0}=a^{0} b^{0}$ since $b \mathcal{H}^{*, \sim} b^{0}$, which implies $b^{0} \leq a^{0}$ and hence $b^{0}=a^{0} b^{0} a^{0}=(a b a)^{0}=(a c a)^{0}=a_{d(\alpha, \beta)}^{0}$, that is, $b \mathcal{H}^{*, \sim} a_{d(\alpha, \beta)}$. Thus by Lemma 10 (ii), $a_{d(\alpha, \beta)}=b$ is required.

2) Since

$$
\left(a^{0}(a x)^{0} a^{0}\right) a^{0}=a^{0}(a x)^{0} a^{0}=a^{0}\left(a^{0}(a x)^{0} a^{0}\right)
$$

and $a^{0}(a x)^{0} a^{0} \mathcal{H}^{*, \sim}\left(a^{0}(a x)^{0} a^{0}\right)^{0}$, we have $\left(a^{0}(a x)^{0} a^{0}\right)^{0} a^{0}=\left(a^{0}(a x)^{0} a^{0}\right)^{0}=a^{0}\left(a^{0}(a x)^{0} a^{0}\right)^{0}$, that is, $a^{0} \geq\left(a^{0}(a x)^{0} a^{0}\right)^{0}$. Also, since $a \in S_{\alpha}$ and $x \in S_{d(\alpha, \beta)}$, we have $a x \in S_{d(\alpha, \beta)}$ and so that $e=\left(a^{0}(a x)^{0} a^{0}\right)^{0}$ by (i). Thereby, we have eax $=\left(a^{0}(a x)^{0} a^{0}\right)^{0} a x=\left(a^{0}(a x)^{0} a^{0}\right)^{0} a^{0}(a x)^{0} a^{0} a x=a x$. Similarly, we have $x a e=x a$. Since $x$ is arbitrarily chosen element in $S_{d(\alpha, \beta)}$, we can particularly choose $x=e$. In this way, we obtain that $e a=a e$ and consequently, by Lemma 1 , we have $(e a)^{0}=\left(e a^{0}\right)^{0}=e$.

Lemma 15 Let $S=\left(Y ; S_{\alpha}\right)$ be a regular $r$-ample semigroup. For any $\alpha \geq \beta$ on $Y$ and $d(\alpha, \beta) \in D(\alpha, \beta)$, define a mapping $\phi_{d(\alpha, \beta)}$ from $S_{\alpha}$ into $S_{d(\alpha, \beta)}$ with a $a \phi_{d(\alpha, \beta)}=a_{d(\alpha, \beta)}$, where $a_{d(\alpha, \beta)}$ is defined in Lemma 14. Write $\Phi_{\alpha, \beta}=\left\{\phi_{d(\alpha, \beta)}: d(\alpha, \beta) \in D(\alpha, \beta)\right\}$. Then

1) $\phi_{d(\alpha, \beta)}$ is a homomorphism.

2) for $\alpha \in Y, \phi_{D(\alpha, \alpha)}$ is the identity homomorphism of $S_{\alpha}$.

3) for $\alpha \geq \beta \geq \gamma$ on $Y, \Phi_{\alpha, \beta} \Phi_{\beta, \gamma} \subseteq \Phi_{\alpha, \gamma}$.

4) for $b \in S_{\beta}$, there exists $\phi_{d(\alpha, \alpha \beta)}^{b} \in \Phi_{\alpha, \alpha \beta}$, for all $a \in S_{\alpha}$,

$$
a b=\left(a \phi_{d(\alpha, \alpha \beta)}^{b}\right)\left(b \phi_{d(\beta, \alpha \beta)}^{a}\right) .
$$

Proof. 1) Following Lemma 14, $\phi_{d(\alpha, \beta)}$ is well defined. For $a, b \in S_{\alpha}$ and $c \in S_{d(\alpha, \beta)}$, by Lemma 14 again, 


$$
\begin{aligned}
\left(a \phi_{d(\alpha, \beta)}\right)\left(b \phi_{d(\alpha, \beta)}\right)=(a c a)^{0} a b(b c b)^{0}= & (a c a)^{0} a b=a b(b c b)^{0} \leq a b \text { and so } \\
(a b) \phi_{d(\alpha, \beta)} & =\left(a \phi_{d(\alpha, \beta)}\right)\left(b \phi_{d(\alpha, \beta)}\right) .
\end{aligned}
$$

2) It follows easily since $S_{\alpha}$ is primitive.

3) We only need to show that for any $d(\alpha, \beta) \in D(\alpha, \beta) d(\beta, \gamma) \in D(\alpha, \gamma), S_{d(\alpha, \beta)} \phi_{d(\beta, \gamma)} \subseteq S_{d(\alpha, \gamma)}$ for some $d(\alpha, \gamma) \in D(\alpha, \gamma)$. Let $a \in S_{\alpha}, b_{1}, b_{2} \in S_{d(\alpha, \beta)}$ and $c \in S_{\gamma}$, we have $\left(a b_{1} a\right)^{0}=\left(a b_{2} a\right)^{0}$ and $b_{1} \phi_{d(\beta, \gamma)}=b_{1}\left(b_{1} c b_{1}\right)^{0}, \quad b_{2} \phi_{d(\beta, \gamma)}=b_{2}\left(b_{2} c b_{2}\right)^{0}$ and so

$$
\left(a\left(b_{1} \phi_{d(\beta, \gamma)}\right) a\right)^{0}=\left(a b_{1}\left(b_{1} c b_{1}\right)^{0} a\right)^{0}=\left(a b_{2}\left(b_{2} c b_{2}\right)^{0} a\right)^{0}=\left(a\left(b_{2} \phi_{d(\beta, \gamma)}\right) a\right)^{0},
$$

which implies $S_{d(\alpha, \beta)} \phi_{d(\beta, \gamma)} \subseteq S_{d(\alpha, \beta)}$ for some $d(\alpha, \gamma) \in D(\alpha, \gamma)$.

4) For $b \in S_{\beta}$, we need to prove that $b \Phi_{\beta, \alpha \beta}=\left\{b \phi_{d(\beta, \alpha \beta)} \mid \forall d(\beta, \alpha \beta) \in D(\beta, \alpha \beta)\right\} \subseteq S_{d(\alpha, \alpha \beta)}$. In fact, it suffices to show that for any $\phi_{d(\beta, \alpha \beta)}$ and $\phi_{d^{\prime}(\beta, \alpha \beta)} \in \Phi_{\beta, \alpha \beta}$, we have $\left(b \phi_{d(\beta, \alpha \beta)}, b \phi_{d^{\prime}(\beta, \alpha \beta)}\right) \in \rho_{\alpha, \alpha \beta}$. For this purpose, we let $x \in S_{d(\beta, \alpha \beta)}$ and $x^{\prime} \in S_{d^{\prime}(\beta, \alpha \beta)}$. Then, by (i), we have $b \phi_{d(\beta, \alpha \beta)}=b(b x b)^{0}$ and $b \phi_{d^{\prime}(\beta, \alpha \beta)}=b\left(b x^{\prime} b\right)^{0}$. Let $a \in S_{\alpha}$, then, because $S_{\alpha \beta}$ is a completely $\mathcal{J}^{*, \sim}$-simple semigroup, and $a b a$, $b \phi_{d(\beta, \alpha \beta)}, b \phi_{d^{\prime}(\beta, \alpha \beta)}$ are elements in $S_{\alpha \beta}$. We obtain that $\left(a b a,(a b a)\left(b \phi_{d(\beta, \alpha \beta)}\right)(a b a)\right) \in \mathcal{H}^{*, \sim}$ and $\left(a b a,(a b a)\left(b \phi_{d^{\prime}(\beta, \alpha \beta)}\right)(a b a)\right) \in \mathcal{H}$. By Lemma 1, we conclude that

$$
\left((a b a)\left(b \phi_{d(\beta, \alpha \beta)}\right)(a b a)\right)^{0}=\left((a b a)\left(b \phi_{d^{\prime}(\beta, \alpha \beta)}\right)(a b a)\right)^{0} .
$$

In other words, $\left((a b a)\left(b(b x b)^{0}\right)(a b a)\right)^{0}=\left((a b a)\left(b\left(b x^{\prime} b\right)^{0}\right)(a b a)\right)^{0}$. Thus, by the regularity of the band $S / \mathcal{H}^{*, \sim}$, we can further simplify the above equality to $\left(a\left(b(b x b)^{0}\right) a\right)^{0}=\left(a\left(b\left(b x^{\prime} b\right)^{0}\right) a\right)^{0}$, that is, $\left(a\left(b \phi_{d(\beta, \alpha \beta)}\right) a\right)^{0}=\left(a\left(b \phi_{d^{\prime}(\beta, \alpha \beta)}\right) a\right)^{0}$. It hence follows, by the definition of $\rho_{\alpha, \alpha \beta}$, that is $\left(b \phi_{d(\beta, \alpha \beta)}, b \phi_{d^{\prime}(\beta, \alpha \beta)}\right) \in \rho_{\alpha, \alpha \beta}$.

Now let $c_{1} \in S_{d^{\prime}(\alpha, \alpha \beta)}, \quad c_{2} \in S_{d^{\prime}(\beta, \alpha \beta)}$. Then $\left(a c_{1} a\right)^{0} \in S_{d^{\prime}(\alpha, \alpha \beta)}$ because $S_{d^{\prime}(\alpha, \alpha \beta)}$ is a $\rho_{\alpha, \alpha \beta}$-equivalence class of $S_{\alpha \beta}$. Now, by (i), $a \phi_{d^{\prime}(\alpha, \alpha \beta)}=\left(a c_{1} a\right)^{0} a$ and $b \phi_{d^{\prime}(\beta, \alpha \beta)}=b\left(b c_{2} b\right)^{0}$ for $\phi_{d^{\prime}(\alpha, \alpha \beta)} \in \Phi_{\alpha, \alpha \beta}$ and $\phi_{d^{\prime}(\beta, \alpha \beta)} \in \Phi_{\beta, \alpha \beta}$. Since we assume that $a \Phi_{\alpha, \alpha \beta} \subseteq S_{d^{\prime}(\beta, \alpha \beta)}$, we have $a \phi_{d^{\prime}(\alpha, \alpha \beta)}=\left(a c_{1} a\right)^{0} a \in S_{d^{\prime}(\beta, \alpha \beta)}$. Similarly, we have $b \phi_{d^{\prime}(\beta, \alpha \beta)} \in S_{d^{\prime}(\alpha, \alpha \beta)} \cap S_{d^{\prime}(\beta, \alpha \beta)}$. Thus, we have $\left(a \phi_{d^{\prime}(\alpha, \alpha \beta)}\right)\left(b \phi_{d^{\prime}(\beta, \alpha \beta)}\right)=\left(a c_{1} a\right)^{0}\left(a b\left(b c_{2} b\right)^{0}\right)=a b\left(b c_{2} b\right)^{0}$ and also

$$
\left(a \phi_{d^{\prime}(\alpha, \alpha \beta)}\right)\left(b \phi_{d^{\prime}(\beta, \alpha \beta)}\right)=\left(\left(a c_{1} a\right)^{0} a b\right)\left(b c_{2} b\right)^{0}=\left(a c_{1} a\right)^{0} a b .
$$

However, by the definition of the natural partial order " $\leq$ " on semigroup $S$, we have $a b \geq\left(a \phi_{d^{\prime}(\alpha, \alpha \beta)}\right)\left(b \phi_{d^{\prime}(\beta, \alpha \beta)}\right)$. On the other hand, because every $S_{\alpha \beta}$ is a completely $\mathcal{J}^{*, \sim}$-simple semigroup, $S_{\alpha \beta}$ is a primitive semigroup. Hence, we obtain that $a b=\left(a \phi_{d^{\prime}(\alpha, \alpha \beta)}\right)\left(b \phi_{d^{\prime}(\beta, \alpha \beta)}\right)$.

Finally, we can easily see that for any $a_{1}, a_{2} \in S_{\alpha}$ and $\beta \in Y$, if $a_{1} \Phi_{\alpha, \alpha \beta}$ and $a_{2} \Phi_{\alpha, \alpha \beta}$ are all subsets of 
the same $\rho_{\beta, \alpha \beta}$-class $S_{d^{\prime}(\beta, \alpha \beta)}$, then $a_{1}$ and $a_{2}$ determine the same mapping $\phi_{d^{\prime}(\beta, \alpha \beta)} \in \Phi_{\beta, \alpha \beta}$ and hence for any $b \in S_{\beta}$, we have $a_{1} b=\left(a_{1} \phi_{d(\alpha, \alpha \beta)}^{b}\right)\left(b \phi_{d^{\prime}(\beta, \alpha \beta)}\right)$ and $a_{2} b=\left(a_{2} \phi_{d(\alpha, \alpha \beta)}^{b}\right)\left(b \phi_{d^{\prime}(\beta, \alpha \beta)}\right)$.

Theorem 16 A $r$-ample semigroup $S$ is a regular $r$-ample semigroup if and only if it is a $G$-strong semilattice of completely $\mathcal{J}^{*, \sim}$-simple semigroups.

Proof. We have already proved the necessity from Lemma 14 and Lemma 15. We now prove the sufficiency part of the theorem. We first show that the Green's $(*, \sim)$-relation $\mathcal{H}^{*, \sim}$ is a congruence on $S$. In fact, if $a \in S_{\alpha}, b \in S_{\beta}$ then by the definition of $G$-strong semilattice and that each $S_{\alpha}$ is a completely $\mathcal{J}^{*, \sim}$ simple semigroup, we see that there exist $\phi_{d(\alpha, \alpha \beta)} \in \Phi_{\alpha, \alpha \beta}$ and $\phi_{d(\beta, \alpha \beta)} \in \Phi_{\beta, \alpha \beta}$ satisfying the following equalities

$$
a b=\left(a \phi_{d(\alpha, \alpha \beta)}\right)\left(b \phi_{d(\beta, \alpha \beta)}\right) \text { and } a^{0} b^{0}=\left(a^{0} \phi_{d(\alpha, \alpha \beta)}\right)\left(b^{0} \phi_{d(\beta, \alpha \beta)}\right)
$$

since $\rho_{\alpha, \beta}$ is a band congruence on $S_{\beta}$. Hence, we deduce that

$$
\begin{aligned}
(a b)^{0} & =\left[\left(a \phi_{d(\alpha, \alpha \beta)}\right)\left(b \phi_{d(\beta, \alpha \beta)}\right)\right]^{0}=\left[\left(a \phi_{d(\alpha, \alpha \beta)}\right)^{0}\left(b \phi_{d(\beta, \alpha \beta)}\right)^{0}\right]^{0} \\
& =\left[\left(a^{0} \phi_{d(\alpha, \alpha \beta)}\right)\left(b^{0} \phi_{d(\beta, \alpha \beta)}\right)\right]^{0}=\left(a^{0} b^{0}\right)^{0} .
\end{aligned}
$$

Now by Lemma $1, \mathcal{H}^{*, \sim}$ is a congruence on $S=\left(Y ; S_{\alpha}\right)$.

To see that $S / \mathcal{H}^{*, \sim}$ is a regular band, by a result of [18], we only need to show that the Green's relations $\mathcal{L}$ and $\mathcal{R}$ are both congruences on $S / \mathcal{H}^{*, \sim}$. We only show that $\mathcal{L}$ is a congruence in $S / \mathcal{H}^{*, \sim}$ as $\mathcal{R}$ is a congruence in $S / \mathcal{H}^{*, \sim}$ which can be proved in a similar fashion. Since $S=\left(Y ; S_{\alpha}\right)$ is a $r$-ample semigroup, we can let $e \mathcal{H}^{*, \sim}, f \mathcal{H}^{*, \sim}$ and $g \mathcal{H}^{*, \sim} \in S / \mathcal{H}^{*, \sim}$, where $e, f \in S_{\alpha} \cap E(S), g \in S_{\beta} \cap E(S)$ with $(e, f) \in \mathcal{L}$. Then,

we have $e f=e$ and $f e=f$. By the definition of $G$-strong semilattice, we can find homomorphisms $\phi_{d(\beta, \alpha \beta)}^{e f}$

and $\phi_{d(\beta, \alpha \beta)}^{f} \in \Phi_{\beta, \alpha \beta}, \quad \phi_{d(\alpha, \alpha \beta)}^{g} \in \Phi_{\alpha, \alpha \beta}$ such that

$$
\begin{aligned}
(\text { gegf }) \mathcal{H}^{*, \sim} & =\{[g(e f)](\text { gf })\} \mathcal{H}^{*, \sim} \\
& =\left\{\left[\left(g \phi_{d(\beta, \alpha \beta)}^{e f}\right)\left((\text { ef }) \phi_{d(\alpha, \alpha \beta)}^{g}\right)\right] \cdot\left[\left(g \phi_{d(\beta, \alpha \beta)}^{f}\right)\left(f \phi_{d(\alpha, \alpha \beta)}^{g}\right)\right]\right\} \mathcal{H}^{*, \sim} \\
& =\left[\left(g \phi_{d(\beta, \alpha \beta)}^{e f}\right)\left(f \phi_{d(\alpha, \alpha \beta)}^{g}\right)\right] \mathcal{H}^{*, \sim}
\end{aligned}
$$

and

$$
(g e) \mathcal{H}^{*, \sim}=[g(e f)] \mathcal{H}^{*, \sim}=\left[\left(g \phi_{d(\beta, \alpha \beta)}^{e f}\right)\left((e f) \phi_{d(\alpha, \alpha \beta)}^{g}\right)\right] \mathcal{H}^{*, \sim}=\left[\left(g \phi_{d(\beta, \alpha \beta)}^{e f}\right)\left(f \phi_{d(\alpha, \alpha \beta)}^{g}\right)\right] \mathcal{H}^{*, \sim} .
$$

Thereby, (gegf $) \mathcal{H}^{*, \sim}=(g e) \mathcal{H}^{*, \sim}$. Analogously, we can also prove that $(g f g e) \mathcal{H}^{*, \sim}=(g f) \mathcal{H}^{*, \sim}$. This proves that $\mathcal{L}$ is left compatible on $S / \mathcal{H}^{*} \sim$. Since $\mathcal{L}$ is always right compatible, we see that $\mathcal{L}$ is a congruence on $S / \mathcal{H}^{*, \sim}$, as required. Dually, $\mathcal{R}$ is also a congruence on $S / \mathcal{H}^{*, \sim}$. Thus by [18] (see II. 3.6 Proposition), $S / \mathcal{H}^{*, \sim}$ is a regular band and hence $S$ is a regular cryptic $r$-ample semigroup. Our proof is completed.

\section{REFERENCES}

[1] A. H. Clifford and G. B. Preston, “The Algebraic Theory of Semigroups,” American Mathematical Society, New York, 1967, pp. 98-120.

[2] A. H. Clifford, “Semigroups Admitting Relative Inverses,” Annals of Mathematics, Vol. 42, 1941, pp. 1037-1049. http://dx.doi.org/10.2307/1968781

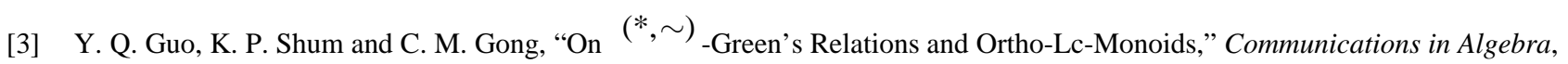
Vol. 39, No. 1, 2011, pp. 5-31. http://dx.doi.org/10.1080/00927870903428247 
[4] J. M. Howie, “Fundamental of Semigroup Theory,” Clarendon Press, Oxford, 1995, pp. 56-73.

[5] M. Petrich and N. R. Reilly, “Completely Regular Semigroups,” John Wiley \& Sons, New York, 1999, pp. 162-197.

[6] M. Petrich, "The Structure of Completely Regular Semigroups,” Transactions of the American Mathematical Society, Vol. 189, 1974, pp. 211-236. http://dx.doi.org/10.1090/S0002-9947-1974-0330331-4

[7] M. Petrich, “Lectures in Semigroups,” Wiley \& Sons Inc., London, 1976, pp. 124-156.

[8] F. Pastijn, “A Representation of a Semigroup by a Semigroup of Matrices over a Group with Zero,” Semigroup Forum, Vol. 10, 1975, pp. 238-249.

[9] J. B. Fountain, “Abundant Semigroups,” Proceedings London Mathematical Society, Vol. 43, No. 3, 1982, pp. $103-129$. http://dx.doi.org/10.1112/plms/s3-44.1.103

[10] M. V. Lawson, “Rees Matrix Semigroups,” Proceedings of the Edinburgh Mathematical Society, Vol. 33, 1990, pp. 23-39.

[11] X. M. Ren, K. P. Shum and Y. Q. Guo, “A Generalized Clifford Theorem of Semigroups,” Science China Mathematics, Vol. 53, No. 4, 2010, pp. 1097-1101. http://dx.doi.org/10.1007/s11425-009-0150-3

[12] X. Z. Kong and K. P. Shum, “On the Structure of Regular Crypto Semigroups,” Communications in Algebra, Vol. 29, No. 6, 2001, pp. 2461-2479. http://dx.doi.org/10.1007/s00233-006-0614-5

[13] X. Z. Kong and Z. L. Yuan, "Normal Crypto H -Abundant Semigroups,” Advances in Mathematics (in Chinese), Vol. 36, No. 5, 2007, pp. 539-545.

[14] X. Z. Kong and Z. L. Yuan, "KG-Strong Semilattice Structure of Regular Orthocryptosemigroups,” Semigroup Fourm, Vol. 73, No. 1, 2006, pp. 95-108. http://dx.doi.org/10.1007/s00233-006-0614-5

[15] X. Z. Kong and K. P. Shum, “A Structure Theorem of Normal $H^{\sim}$-Cryptographs,” Publicationes Mathematicae, Vol. 72, No. 3-4, 2008, pp. 335-346.

[16] X. Z. Kong, Z. L. Yuan and K. P. Shum, “ $H^{\#}$-Abundant Semigroups and $H^{\#}$-Cryptographs,” Algebra Collections, Vol. 15, No. 4, 2008, pp. 653-666.

[17] K. P. Shum, L. Du and Y. Q. Guo, “Green’s Relations and Their Generalizations on Semigroups,” Discussiones Mathematicae —General Algebra and Applications, Vol. 30, No. 1, 2010, pp. 71-89. http://dx.doi.org/10.7151/dmgaa.1163

[18] X. D. Tang, “On a Theorem of C-Wrpp Semigroups,” Communications in Algebra, Vol. 25, No. 5, 1997, pp. 1499-1504. http://dx.doi.org/10.1080/00927879708825931

[19] G. M. S. Gomes and V. Gould, "Proper Weakly Left Ample Semigroups,” International Journal of Algebra and Computation, Vol. 9, No. 6, 1999, pp. 721-739. http://dx.doi.org/10.1142/S0218196799000412 